Article

\title{
Electrostatic Potential and a Simple Extended Electric Dipole Model of Hydrogen Fluoride as Probes of Non-Bonding Electron Pairs in the Cyclic Ethers 2,5-Dihydrofuran, Oxetane and Oxirane
}

\author{
J. Grant Hill ${ }^{1}$ (i) and Anthony C. Legon ${ }^{2, *}$ \\ 1 Department of Chemistry, University of Sheffield, Sheffield S3 7HF, UK; grant.hill@sheffield.ac.uk \\ 2 School of Chemistry, University of Bristol, Cantocks Close, Bristol BS8 1TS, UK \\ * Correspondence: a.c.legon@bristol.ac.uk
}

Academic Editors: Peter Politzer and Jane S. Murray

Received: 7 July 2017; Accepted: 22 August 2017; Published: 25 August 2017

\begin{abstract}
The electrostatic potential near to the oxygen atom in each of the cyclic ethers 2,5-dihydrofuran, oxetane and oxirane has been calculated by using a distributed multipole analysis (DMA) of each molecule. The electrostatic potential energy $V(\phi)$ of a unit non-perturbing positive charge was calculated (via the DMA of the cyclic ether molecule) as a function of the angle $\phi$ between the $C_{2}$ axis of the cyclic ether and a vector of length $r$ from the $\mathrm{O}$ atom to the unit charge. The resulting potential energy functions each has two equivalent minima. The angles $\phi_{\min }$ at the minima are compared with the angles $\phi_{0}$ and $\phi_{\mathrm{e}}$ made by the $\mathrm{O} \cdots \mathrm{H}$ bond with the $C_{2}$ axes in the cyclic ether...HF complexes, as determined by rotational spectroscopy and ab initio calculations at the CCSD(T)-F12c/cc-pVTZ-F12 level of theory, respectively. An electrostatic model of cyclic ether $\cdot$ HF complexes in which the DMA of the cyclic ether interacts with a simple extended electric dipole representation of HF is also used to calculate the variation of the potential energy $V_{\mathrm{HF}}(\phi)$ of the HF molecule with $\phi$. The angles $\phi_{\min }$ generated by this model are also compared with $\phi_{0}$ and $\phi_{\mathrm{e}}$. The extent to which the electrostatic potential and the extended electric dipole HF model can be used as probes for the directions of non-bonding electron pairs carried by $\mathrm{O}$ in these cyclic ethers is discussed.
\end{abstract}

Keywords: hydrogen bond; noncovalent interactions; electrostatic potentials; distributed multipole analysis; nonbonding-pairs

\section{Introduction}

The hydrogen bond and the halogen bond are the best-known of non-covalent interactions and have recently been defined by IUPAC Working Parties [1,2]. Several other types of non-covalent interaction have been named and described [3]. In each case, a simple model can be applied to account for the bond, namely that an electrophilic region of the molecule acting as a Lewis acid A (e.g., the hydrogen atom participating in a hydrogen bond or a halogen atom in a halogen bond) interacts with a nucleophilic region of a molecule acting as a Lewis base B (e.g., a non-bonding electron pair or a $\pi$-bonding electron pair). The electrophilic region has been referred to as either a $\sigma$-hole or $\pi$-hole $[4,5]$.

In this article, we shall focus attention on the nucleophilic region of the Lewis base B that accepts the hydrogen atom on hydrogen bond formation, particularly the non-bonding electron pairs (n-pairs) of B. The geometries of simple hydrogen-bonded complexes B $\cdots$ HX (where X is a halogen atom, a $\mathrm{CN}$ group or $\mathrm{C} \equiv \mathrm{CH}$ ) isolated in the gas phase at very low pressure have been extensively investigated by microwave and infrared spectroscopy over many years. The systematic variation of $\mathrm{B}$ led to a set of 
simple rules $[6,7]$ for predicting the angular geometry (i.e., the relative orientation of B and HX) of a complex B $\cdots$ HX. The rules are as follows:

The gas-phase equilibrium angular geometry of a complex $\mathrm{B} \cdots \mathrm{HX}$ can be obtained by assuming that:

(i) the axis of the HX molecule coincides with the supposed axis of a non-bonding electron pair of $B$, as conventionally envisaged or, if $B$ has no nonbonding electron pairs but has $\pi$-bonding electron pairs, (ii) the axis of the HX molecule intersects the internuclear axis of the atoms forming the $\pi$-bond and is perpendicular to the plane of symmetry of the $\pi$-bond. Rule (i) is definitive when $B$ has both non-bonding and $\pi$-bonding pairs.

These rules are electrostatic in origin, a conclusion that was tested by examining the angular dependence of the electrostatic potential in the vicinity of the acceptor atom/centre of B [7] and by the success of the Buckingham-Fowler electrostatic model for hydrogen-bonded complexes [8]. Figure 1 shows how the electrostatic potential $V(\phi)$ (i.e., the potential energy of a unit, non-perturbing positive charge) varies with the angle $\phi$ (defined in Figure 1 ) at a fixed distance $(1.74 \AA$, the experimental $\mathrm{O} \cdots \mathrm{H}$ distance in $\mathrm{H}_{2} \mathrm{O} \cdots \mathrm{HF}$ ) from the $\mathrm{O}$ atom in $\mathrm{H}_{2} \mathrm{O}$ [7]. The potential $V(\phi)$ has two equivalent minima at $\sim \pm 30^{\circ}$, separated by a very low barrier to the planar arrangement at $\phi=0^{\circ}$.

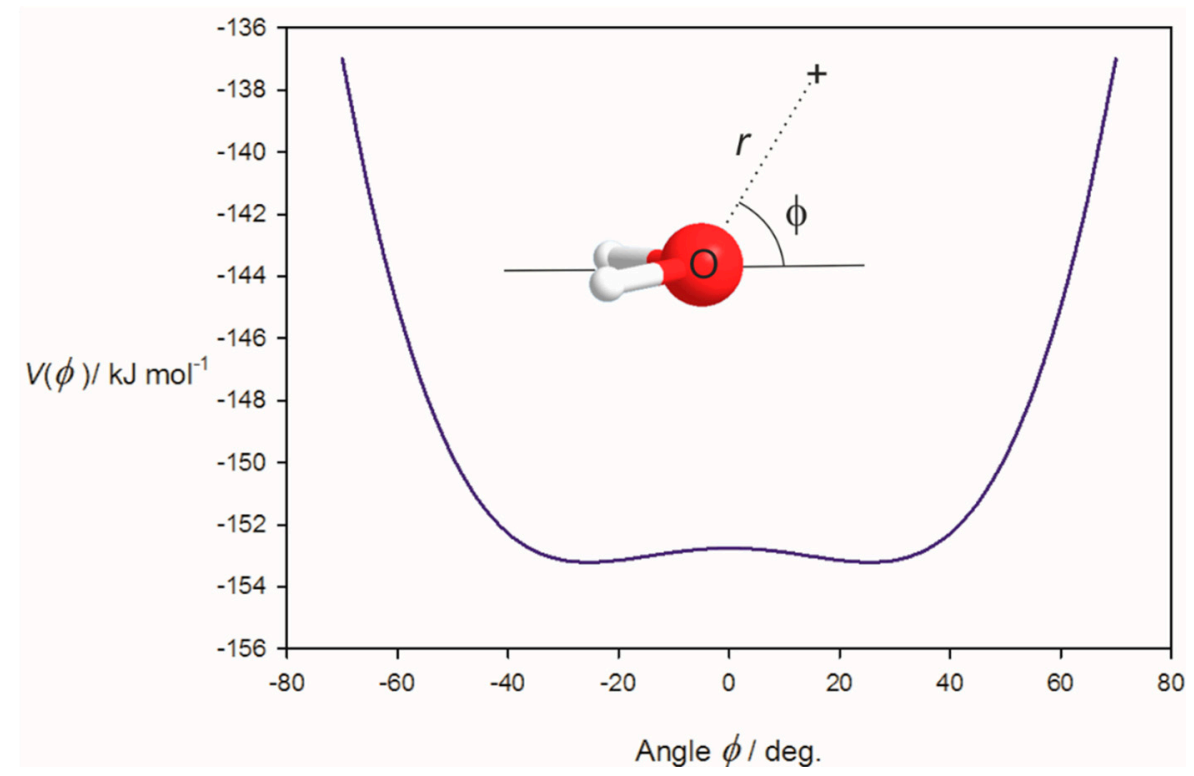

Figure 1. Variation of the electrostatic potential $V(\phi)$ with the angle $\phi$ at distance $r$ from the $\mathrm{O}$ atom in $\mathrm{H}_{2} \mathrm{O}$. The angle $\phi$ is that made by $r$ with the $C_{2}$ axis of the molecule.

A detailed analysis of the rotational spectrum of $\mathrm{H}_{2} \mathrm{O} \cdots \mathrm{HF}$ [9] in the ground state and in excited states associated with the out-of-plane, low frequency bending mode of the molecule led to a similar experimental potential energy function $V(\phi)$ versus $\phi$, with $\phi_{\mathrm{e}}= \pm 46(8)^{\circ}$. An even better match with the experimentally determined function $V_{\mathrm{HF}}(\phi)$ is obtained if an extended electric dipole model of HF is used. This was constructed by replacing the non-perturbing unit positive charge by two non-perturbing charges $q=+0.540 e$ and $-0.540 e$ separated by the $r_{0}$ bond length $0.9256 \AA$ of HF (calculated from its ground-state rotational constant [10]), as suggested by the distributed multipole model of HF given in Ref. [8]. The result is the curve shown in Figure 2. Note that now $\phi_{\mathrm{e}}= \pm 55^{\circ}$. 


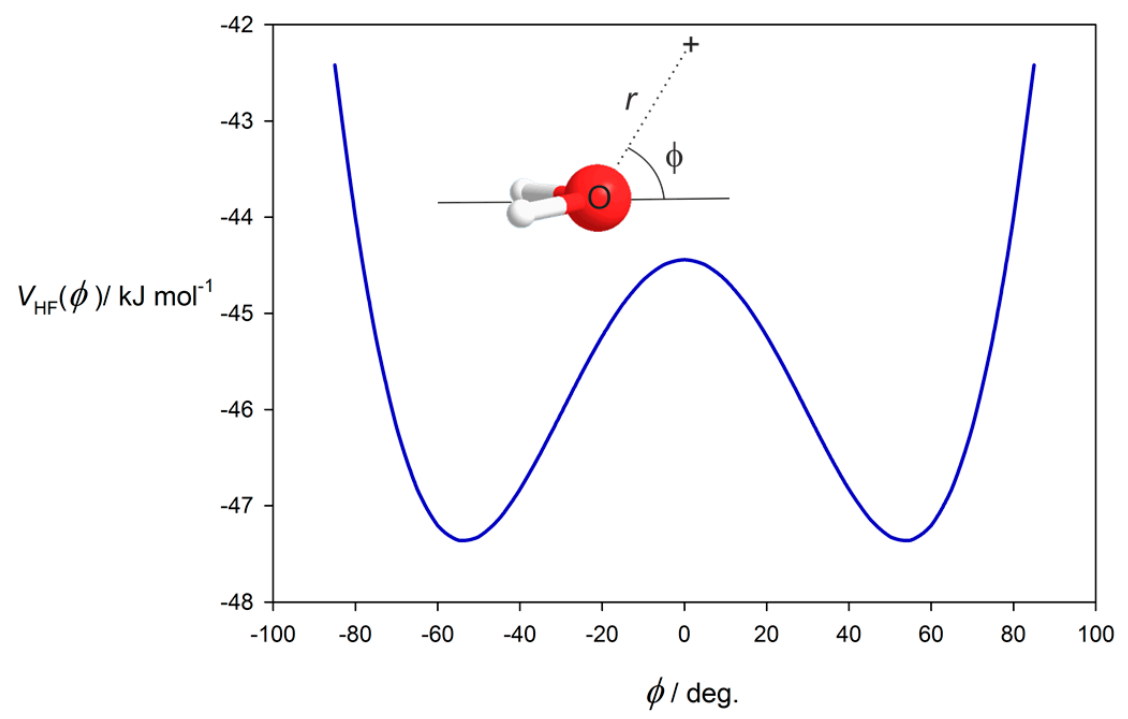

Figure 2. Variation of the electrostatic potential energy $V_{\mathrm{HF}}(\phi)$ of $\mathrm{HF}$ as a function of the angle $\phi$ made by the vector $r$ with the $\mathrm{C}_{2}$ axis of the $\mathrm{H}_{2} \mathrm{O}$ molecule. $\mathrm{HF}$ is treated as an extended electric dipole (see text for details). $r$ is the distance of the $\mathrm{H}$ atom of $\mathrm{HF}$ from the $\mathrm{O}$ atom in $\mathrm{H}_{2} \mathrm{O}$. The $\mathrm{O} \cdots \mathrm{H}-\mathrm{F}$ nuclei are assumed collinear in the model.

The success of the extended electric dipole moment model of $\mathrm{HF}$ in reproducing the experimentally determined function $V(\phi)$ versus $\phi$ for $\mathrm{H}_{2} \mathrm{O} \cdots \mathrm{HF}$ and for predicting $\phi_{\mathrm{e}}$ values for $\mathrm{H}_{2} \mathrm{~S}$ and $\mathrm{H}_{2} \mathrm{CO}$ [7] led to the proposal of the following corollary to the rules: As long as the hydrogen bond in $B \cdots H F$ is sufficiently weak that the electric charge distributions of $B$ and HF are essentially unperturbed, the HF axis acts as a probe for the direction of a nonbonding (or $\pi$-bonding) pair on $B$.

In the original investigations of the rotational spectra of complexes B $\cdots$ HF formed in equilibrium gas mixtures of B and HF [11-13], three Lewis bases were chosen to test whether HF does indeed act as a probe for n-pair directions. The Lewis bases were the following cyclic ethers: 2,5-dihydrofuran, oxetane and oxirane, the geometry of each of which is shown, drawn to scale, in Figure 3 . The reason for this choice is that each has a planar geometry (albeit effectively planar in the zero-point state in the case of oxetane, which is slightly puckered at equilibrium but has a very low barrier to the planar form lying well below the zero-point level) and therefore the $\mathrm{O}$ atom in each should carry two equivalent $n$-pairs (shown in the exaggerated form used by chemists for oxirane in Figure 3 ). However, the internal ring angle $\mathrm{C}-\mathrm{O}-\mathrm{C}$ decreases from $\sim 108^{\circ}$ in 2,5-dihydrofuran [14], through $\sim 90^{\circ}$ in oxetane [15] to $\sim 60^{\circ}$ in oxirane [16] and it was expected that the angle between the n-pairs should increase significantly along the series. In each of the three complexes, $\mathrm{HF}$ forms a hydrogen bond to the $\mathrm{O}$ atom and there is a pyramidal configuration at $\mathrm{O}$ formed by the two equivalent $\mathrm{C}-\mathrm{O}$ bonds and the $\mathrm{O} \cdots \mathrm{H}$ hydrogen bond.
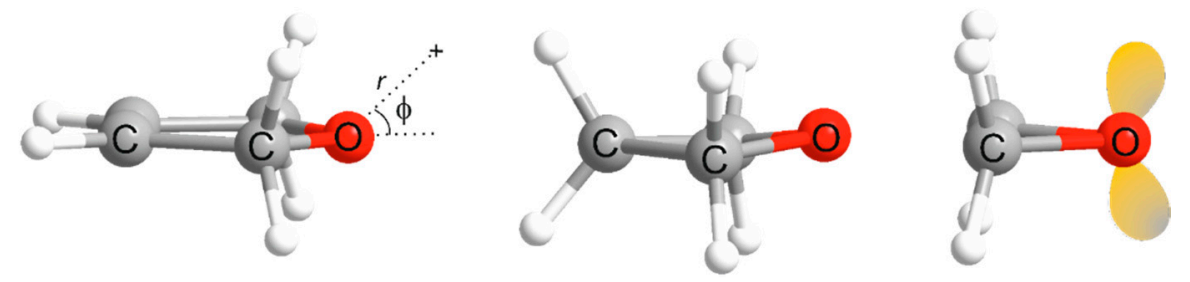

Figure 3. The cyclic ethers 2,5-dihydrofuran, oxetane and oxirane. The n-pairs on $\mathrm{O}$ in oxirane are drawn in the exaggerated form often used by chemists. 
Figure 4 shows the equilibrium geometry (drawn to scale) of each of 2,5-dihydrofuran $\cdots \mathrm{HF}$, oxetane...HF and oxirane..HF as obtained by ab initio calculations carried out at the CCSD(T)-F12c/cc-pVTZ-F12 level of theory. The quantity of immediate interest here is the angle $\phi_{\mathrm{e}}$ made by the $\mathrm{O} \cdots \mathrm{H}$ hydrogen bond with the $C_{2}$ symmetry axis of the cyclic ether. The values were $\phi_{\mathrm{e}}=48.2^{\circ}, 57.4^{\circ}$ and $73.1^{\circ}$, respectively for 2,5 -dihydrofuran $\cdots \mathrm{HF}$, oxetane $\cdots$ HF and oxirane $\cdots \mathrm{HF}$. The corresponding experimental (zero-point) values $\phi_{0}$, determined from rotational spectroscopy by assuming unperturbed component geometries with the $\mathrm{O} \cdots \mathrm{H}-\mathrm{F}$ nuclei collinear, were $48.48(1)^{\circ}$ [11], $57.9^{\circ}[12]$ and $71.8^{\circ}$ [13], respectively. Later re-investigations of these complexes by the more sensitive technique of pulsed-jet, Fourier-transform microwave spectroscopy allowed the linearity constraint to be relaxed and gave $46.34(4)^{\circ}[17], 54.5^{\circ}$ [18] and 72.0(4) ${ }^{\circ}$ [19], respectively, for the angles $\phi_{0}$. Clearly, there is good agreement between theory and experiment for this important quantity. Thus, if the corollary to the rules is valid, both the ab initio calculated angles $2 \phi_{\mathrm{e}}$ and the experimental angles $2 \phi_{0}$ between the two n-pairs do appear to increase along the series. The angular deviation $\theta$ of the hydrogen bond from linearity is found to be small in all three cases by the ab initio calculations (see Table 1), but is a difficult quantity to determine accurately from experiment.
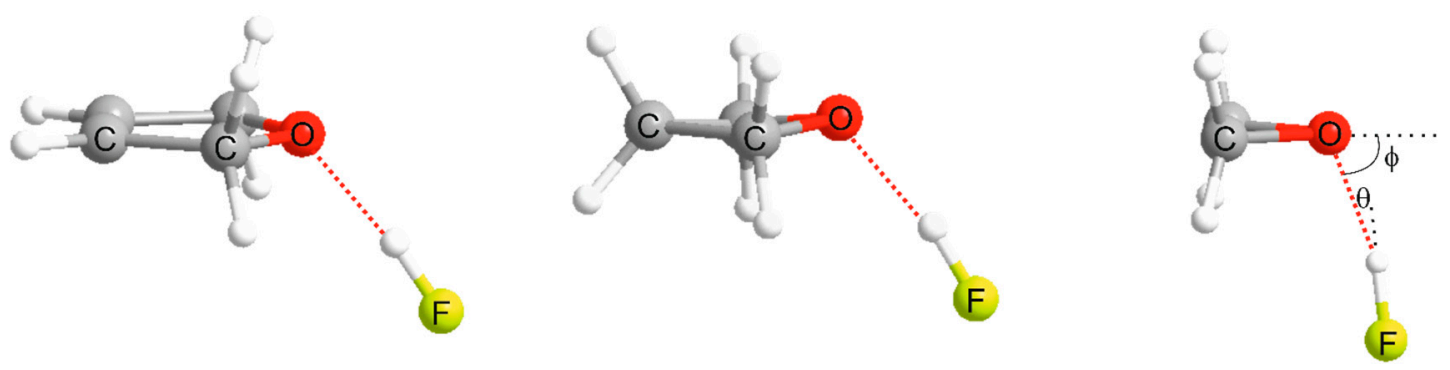

Figure 4. Equilibrium geometries of complexes of 2,5-dihydrofuran, oxetane and oxirane with HF from calculations at the CCSD(T)-F12c/cc-pVTZ-F12 level of theory. The equilibrium angle $\phi$ is indicated for the oxirane complex. The angle $\theta$ defines the deviation of the hydrogen bond nuclei $\mathrm{O} \cdots \mathrm{H}-\mathrm{F}$ from collinearity.

As stated earlier, the rules for predicting angular geometries are electrostatic in origin in the sense that unperturbed electric charge distributions are assumed to define the angular geometry assumed by the interacting pair of molecules, i.e., the effects of polarisation of one molecule by the other and of charge transfer are assumed negligible. In fact, recently there has been considerable controversy and much discussion about the role of polarization [20] and that of charge transfer [21,22] in determining angular geometry in complexes formed through the various types of non-covalent interaction. The effects of the interplay between charge transfer and rehybridization in the two subunits brought about by non-covalent interaction have also been discussed [23]. A comprehensive account by Kolár and Hobza [24] of computational modelling of halogen bonding and other $\sigma$-hole interactions also discusses these topics and includes a more recent use of hydrogen fluoride as a probe for intermolecular interaction than that first described in Refs. [6,7].

The purpose of this article is to examine the electrostatic potential $V(\phi)$ as a function of the angle $\phi$ in the region of the $\mathrm{O}$ atom of each of the three cyclic ethers shown in Figure 3 and also the potential energy of the extended dipole model of HF as a function of $\phi$. The electric charge distribution of each cyclic ether used to calculate the electrostatic potential around $\mathrm{O}$ at a fixed distance was obtained from a distributed multipole analysis (DMA) [25] based on CCSD/cc-pV5Z optimized wavefunctions. Do the values of the angles $2 \phi_{\min }$ between the minima of these potential functions agree (1) with the values of $2 \phi_{0}$ from the microwave spectroscopic investigations of the cyclic ether $\cdots$ HF complexes and (2) with the values of $2 \phi_{\mathrm{e}}$ obtained for the same set of complexes from ab initio calculations conducted at the CCSD(T)-F12c/cc-pVTZ-F12 level of theory? The latter comparison is probably more suitable because both quantities refer to the equilibrium properties of the complex, while the values 
$2 \phi_{0}$ obtained from the rotational spectra are rather complicated averages over the large amplitude zero-point motions. The ab initio calculations also give more accurate values of the angular deviation $\theta$ of the $\mathrm{O} \cdots \mathrm{H}-\mathrm{F}$ nuclei from collinearity.

\section{Computational Details}

Geometries of isolated molecules and the interacting complexes were optimised using the explicitly correlated CCSD(T)-F12c method [26] in the MOLPRO system of ab initio programs [27,28]. The correlation consistent cc-pVTZ-F12 basis set was used [29], which includes diffuse functions in the lower angular momentum shells. Density fitting of the Fock and exchange matrices used the cc-pVTZ/JKFit auxiliary basis [30], while other two electron integrals were density fitted using the cc-pVTZ-F12/MP2Fit set [31]. The many electron integrals occurring in F12 theory were evaluated using the resolution-of-the-identity with the complementary auxiliary basis set (CABS) approach and the cc-pVTZ-F12/OptRI set [32,33]. The geminal Slater exponent was set to $1.0 \mathrm{a}_{0}{ }^{-1}$ throughout. Full details of the optimized geometries are available as Supplementary Materials.

Distributed multipole analyses [25] were carried out on the optimised geometries of the isolated molecules and the detailed results are available as Supplementary Materials. The first-order density matrix required for this analysis was obtained at the CCSD/cc-pV5Z level with the orbital relaxation contribution $[34,35]$. Perturbative triple excitations would not affect the density matrix as this method does not calculate amplitudes for the triple excitations. Molecular electrostatic potential maps (MESPs) were generated at the MP2 / 6-311++ $G^{* *}$ level of theory [36] in the Spartan package [37] not only with the now-conventional isodensity surface of $0.001 \mathrm{e} \mathrm{bohr}^{-3}$ but also with considerably larger isodensities.

\section{Results and Discussion}

\subsection{Electrostatic Potential $V(\phi)$ as a Function of the Angle $\phi$}

The electrostatic potential energy $V(\phi)$ of a unit positive, non-perturbing charge as a function of the angle $\phi$ (see Figure 4 for the definition of $\phi$ ) at fixed distance $r$ from the O nucleus in each of 2,5-dihydrofuran is displayed in Figure 5, while those for oxetane and oxirane are both in Figure 6. The distance $r=1.6 \AA$ was chosen for oxirane and oxetane (Figure 6) because this value is close to the experimental distances $r(\mathrm{O} \cdots \mathrm{H})[11-13,17-19]$ for their complexes with $\mathrm{HF}$ and to the ab initio versions presented here. The functions $V(\phi)$ at this distance both exhibit two equivalent minima, with $\phi_{\min }=\sim \pm 50^{\circ}$ and $\sim \pm 60^{\circ}$ for oxetane and oxirane, respectively, and both show substantial energy barriers to a planar configuration at $\mathrm{O}$. These results are consistent with the various experimental investigations of the complexes via their rotational spectra. Thus, oxirane $\cdots$ HF and oxetane $\cdots$ HF both have a permanently pyramidal configuration at $\mathrm{O}$ in the zero-point energy state, with no evidence of inversion, and have angles $\phi_{0}=72.0(4)^{\circ}$ and $54.50^{\circ}$, respectively [11,12,18,19].

Although the experimental hydrogen bond length is also $r(\mathrm{O} \cdots \mathrm{H}) \approx 1.6 \AA$ for 2,5-dihydrofuran $\cdots \mathrm{HF}, V(\phi)$ as a function of $\phi$ for 2,5-dihydrofuran has only a rather flat single minimum at $\phi=0^{\circ}$ (upper red curve in Figure 5) at this distance. When $r$ is reduced in small steps, a double minimum starts to appear at $1.3 \AA$ (see the orange curve in Figure 5). At $r=1.0 \AA$ (blue curve in Figure 5), the energy barrier at $\phi=0^{\circ}$ is substantial and the minima occur at $\phi_{\min }=\sim \pm 50^{\circ}$. Similar behaviour was reported in Ref. [7] and here (Figure 1) for the variation of $V(\phi)$ with $r$ for the $\mathrm{H}_{2} \mathrm{O}$ molecule, that is, when $r$ was set to the experimental hydrogen bond length $r(\mathrm{O} \cdots \mathrm{H})=1.74 \AA$ of $\mathrm{H}_{2} \mathrm{O} \cdots \mathrm{HF}$, both the height of the potential barrier at $\phi=0^{\circ}\left(0.8 \mathrm{~kJ} \mathrm{~mol}^{-1}\right)$ and $\phi_{\min }=\sim \pm 30^{\circ}$ were underestimates when compared with the experimental potential energy function governing inversion of the configuration at $\mathrm{O}$ in $\mathrm{H}_{2} \mathrm{O} \cdots \mathrm{HF}$ [9], which has a potential energy barrier of $1.5 \mathrm{~kJ} \mathrm{~mol}^{-1}$ to the planar molecule and $\phi_{\min }= \pm 46^{\circ}$. In a recent detailed study, Alonso and co-workers [17] reinvestigated the rotational spectrum of 2,5-dihydrofuran $\cdots$ HF at very high resolution and discovered a small doubling in transitions which they attributed to the motion that inverts the configuration at $\mathrm{O}$ and from which they determined the barrier to the inversion motion in question to be $\sim 1.2 \mathrm{~kJ} \mathrm{~mol}^{-1}$ 
and $\phi_{\min }= \pm 46^{\circ}$. An ab initio calculation of the inversion potential at the MP2/6-311+G(d,p) level confirmed their experimental result. Thus, the electrostatic potential $V(\phi)$ calculated with a non-perturbing unit positive charge at the experimental $\mathrm{O} \cdots \mathrm{H}$ distance appears to underestimate the angle between the n-pairs for both water and 2,5-dihydrofuran. The reason for this was discussed in Ref. [7] and is as follows.

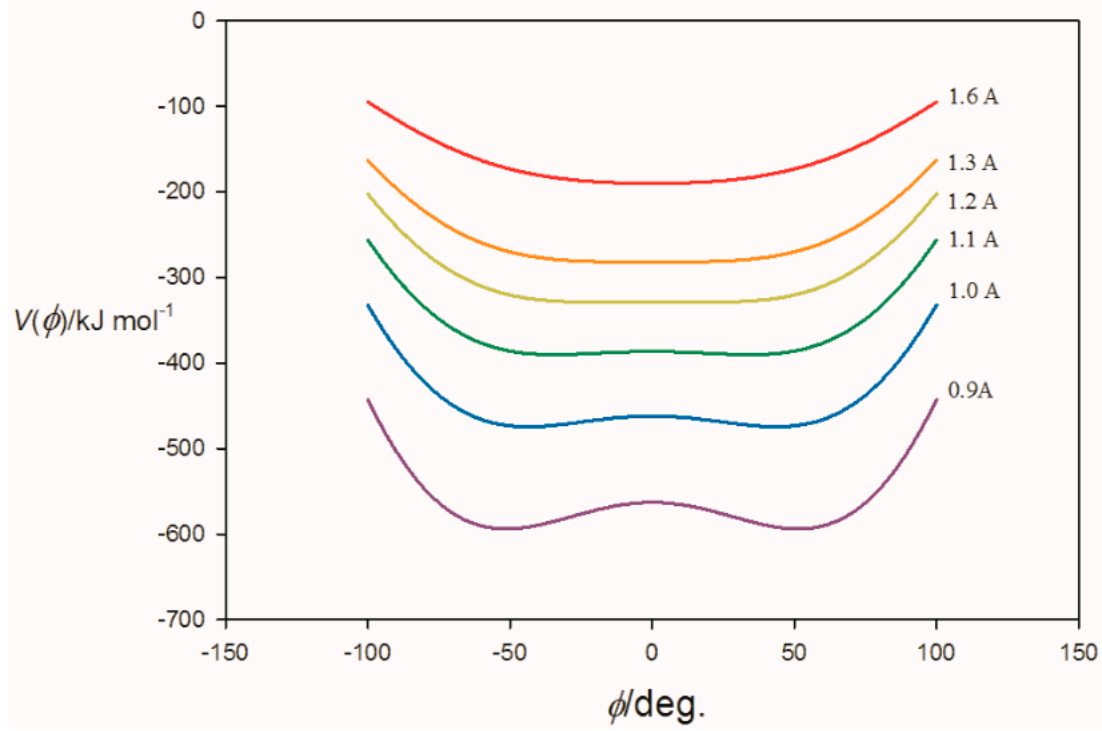

Figure 5. Variation of the electrostatic potential $V(\phi)$ with angle $\phi$ at the indicated distances from the $\mathrm{O}$ atom in 2,5-dihydrofuran.

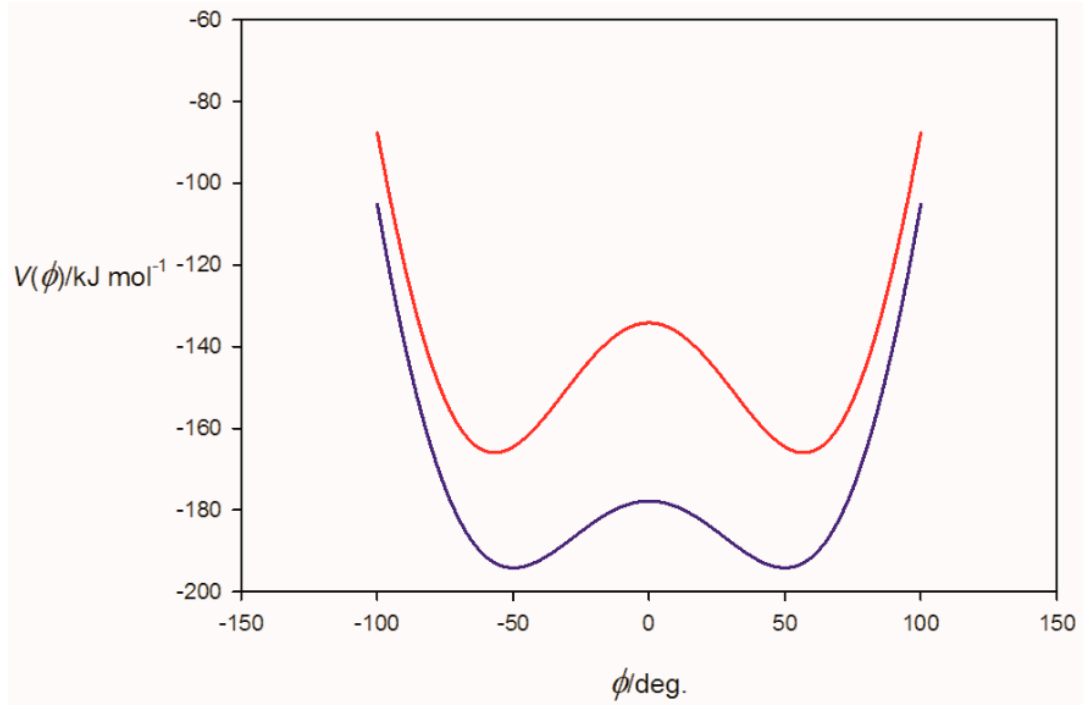

Figure 6. Variation of the electrostatic potential $V(\phi)$ with angle $\phi$ at the distance $r=1.6 \AA$ from the O atom of oxetane (blue curve) and oxirane (red curve).

The electrostatic potential at any point near to a n-pair is determined only in part by the n-pair in question. It also has contributions from any partial positive charge on the protons, carbon atoms, etc. of the water or cyclic ether molecule and from the negative charge of the other n-pair. This partial positive charge acts to decrease the potential of the unit point positive charge for a given angle and becomes more effective as $\phi$ increases. On the other hand, the effect of the other n-pair is to change $V(\phi)$ more slowly than expected as $\phi$ is reduced from $\phi_{\min }$ to zero. Hence, $\phi_{\min }$ will always be less than 
that expected for an isolated nonbonding pair. This effect is more serious in $\mathrm{H}_{2} \mathrm{O}$ and 2,5-dihydrofuran than in oxirane and oxetane because the angle between the nonbonding pairs is smaller in the first two.

Another popular approach to electrostatic potential is the molecular electrostatic surface potential (or MESP). This is the electrostatic potential at the isosurface for which the electron density has a constant value. It is conventional to use an electron density of $0.001 e \mathrm{bohr}^{-3}$ to define the isosurface, which then contains $99.3 \%$ of the electron density. Figure 7 shows the MESPs at $0.001 e \mathrm{bohr}^{-3}$ for the three cyclic ethers under discussion, each viewed along the $C_{2}$ axis with $\mathrm{O}$ nearest the viewer. The calculations were carried out with the SPARTAN package [37] and used the MP2/6-311++ $G^{* *}$ level of theory. The deepest red colour corresponds to an electrostatic potential at the surface of $-200 \mathrm{~kJ} \mathrm{~mol}^{-1}$ and delineates the most nucleophilic region of the molecule. A blue colour corresponds to an electrostatic potential of $+200 \mathrm{~kJ} \mathrm{~mol}^{-1}$ and is the most electrophilic region (not visible). There is clearly a significant nucleophilic region on the isosurface near $\mathrm{O}$ in each molecule. This corresponds to the two n-pairs carried by $\mathrm{O}$, but we note that, at the resolution available in Figure 7, the two n-pairs appear to have merged into a single feature in each case. If a surface of electron density larger than $0.001 \mathrm{ebohr}^{-3}$ is chosen the electrophilic regions (blue) are exaggerated but it is then possible to resolve the two n-pairs, as can be seen in Figure 8. For oxirane, for which the angle between the n-pairs is greatest, the first resolution can be observed at $0.01 \mathrm{e} \mathrm{bohr}{ }^{-3}$ (95.6\% of the electron density), while for 2,5-dihydrofuran, which has the smallest inter n- pair angle, the resolution is only just perceptible even at $0.055 \mathrm{ebohr}^{-3}(84.3 \%)$. Oxetane is an intermediate case, requiring $0.04 \mathrm{e} \mathrm{bohr} \mathrm{r}^{-3}(87.6 \%)$. These observations about the MESPs agree with the conclusions suggested by the $V(\phi)$ versus $\phi$ curves shown in Figure 5, namely that the n-pairs on $\mathrm{O}$ in 2,5-dihydrofuran cannot be identified as separate entities until the distance from $\mathrm{O}$ is much smaller than is required for oxirane.

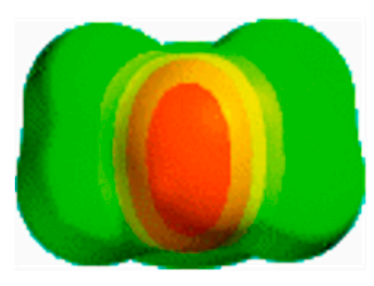

(a)

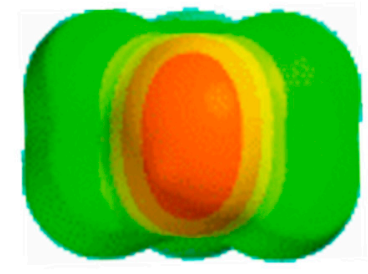

(b)

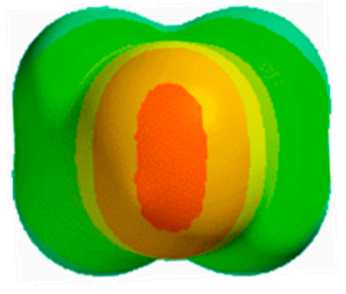

(c)

Figure 7. Molecular electrostatic potential at the $0.001 e$ bohr ${ }^{-3}$ isodensity surfaces of (a) 2,5dihydrofuran, (b) oxetane and (c) oxirane.

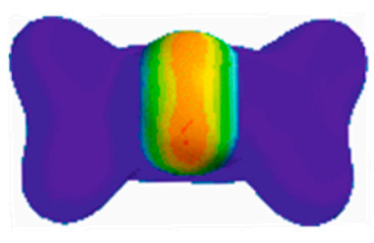

(a)

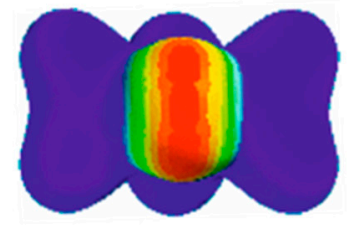

(b)

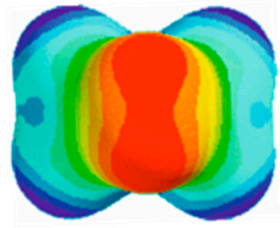

(c)

Figure 8. Molecular electrostatic potential surfaces at the isodensities $0.010 e \mathrm{bohr}^{-3}, 0.040 e \mathrm{bohr}^{-3}$ and $0.055 \mathrm{ebohr}^{-3}$ in (a) 2,5-dihydrofuran, (b) oxetane and (c) oxirane, respectively.

Another way to test the explanation given earlier is to remove the multipoles from all atoms but $\mathrm{O}$ in 2,5-dihydrofuran. Then, there can be no swamping effect on the oxygen n-pairs arising from the charge distributions associated with $\mathrm{H}$ and $\mathrm{C}$ atoms in the rest of the molecule. The result of so doing is shown in Figure 9, where we see that a double minimum function $V(\phi)$ is now obvious for 
2,5-dihydrofuran, even though the distance of the non-perturbing point charge from $\mathrm{O}$ is $r=1.6 \AA$. Note that now $\phi_{\min }=54^{\circ}$, precisely half the tetrahedral angle, as expected from the COC ring angle of $\sim 108^{\circ}$ of 2,5-dihydrofuran. Thus, the potential energy curves in Figure 9 show nicely that the angle between the two minima (and therefore presumably the angle between the n-pairs) increases as the number of carbon atoms in the ring and the internal ring angle at $\mathrm{O}$ are reduced. Also in Figure 8 are the corresponding functions at $r=1.6 \AA$ that arise when multipoles are removed from all atoms but $\mathrm{O}$ in oxetane and oxirane.

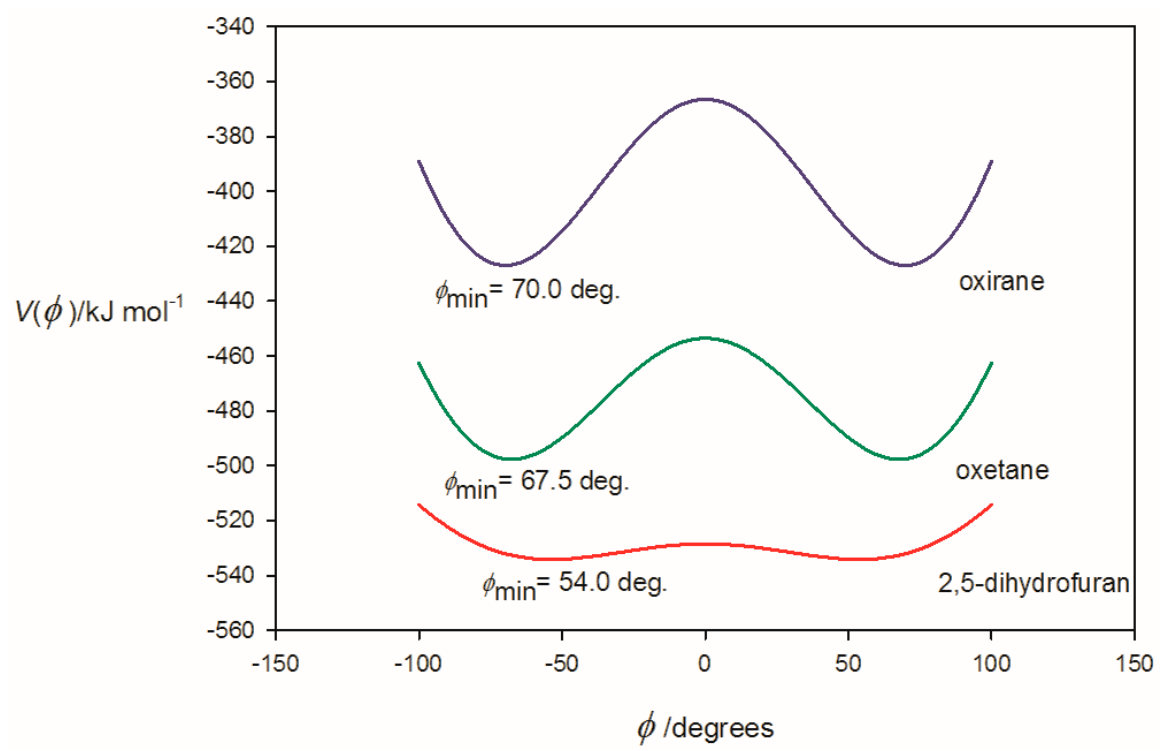

Figure 9. Variation of the electrostatic potential $V(\phi)$ with angle $\phi$ at a distance $r=1.6 \AA$ from the O atom in each of 2,5-dihydrofuran, oxetane and oxirane when the multipoles on all atoms but oxygen are set to zero.

\subsection{Potential Energy of an Extended Electric Dipole Model of HF as a Function of Angle $\phi$}

As mentioned in the Introduction, the success of a simple extended electric dipole model of HF in predicting the angular geometries of $\mathrm{H}_{2} \mathrm{O} \cdots \mathrm{HF}$ and $\mathrm{H}_{2} \mathrm{~S} \cdots \mathrm{HF}$ gave rise to a proposal that the HF molecules acts as a probe of n-pairs carried by Lewis bases, assuming the hydrogen bond is weak enough that the component molecules are not significantly perturbed by the interaction. We now test this proposal for the three Lewis bases 2,5-dihydrofuran, oxetane and oxirane.

The model used for the charge distribution of the HF molecule consists of a pair of charges $0.540 e$ and $-0.540 e$ located on the $\mathrm{H}$ and $\mathrm{F}$ atoms, respectively, and separated by the $r_{0}$ bond length $(0.9256 \AA)$ of HF. This model is the zeroth-order version of the DMA of hydrogen fluoride given in ref. [8]. The extended electric dipole is then assumed to lie on a line that makes an angle $\phi$ with the $C_{2}$ axis of the cyclic ether, so that the atoms lie in the order $\mathrm{O} \cdots \mathrm{H}-\mathrm{F}$ and are collinear. The distance of $\mathrm{H}$ from $\mathrm{O}$ is $r$. The electrostatic potentials $V_{\mathrm{H}}(\phi)$ and $V_{\mathrm{F}}(\phi)$ of the two charges that constitute the model of HF were next calculated using the DMA of the cyclic ether. The electrostatic potential energy of the HF molecule is then given by $V_{\mathrm{HF}}(\phi)=V_{\mathrm{H}}(\phi)+V_{\mathrm{F}}(\phi)$.

The plots of $V_{\mathrm{HF}}(\phi)$ versus $\phi$ when the cyclic ether is 2,5-dihydrofuran are shown in Figure 10 when $r$ is taken from $1.0 \AA$ to $1.6 \AA$ in $0.1 \AA$ steps. Unlike the simple electrostatic potential curves in Figure 5 , a double minimum is now evident in each of the curves, albeit with tiny barriers of 0.3 and $0.05 \mathrm{~kJ} \mathrm{~mol}^{-1}$ at 1.5 and $1.6 \AA$, respectively. It was noted earlier that the potential energy function determined by Alonso and co-workers [17] for 2,5-dihydrofuran $\cdots$ HF has a barrier to the planar configuration at $\mathrm{O}$ of only $\approx 1 \mathrm{~kJ} \mathrm{~mol}^{-1}$ and minima at $\phi= \pm 46^{\circ}$. The extended dipole model of HF interacting with 2,5-dihydrofuran gives curves that are consistent with the experimental conclusion, but with minima separated by smaller values of $2 \phi_{\min }$, for reasons discussed in Section 3.1 by reference 
to Figure 5. We note that as $r$ decreases, $2 \phi_{\min }$ increases and achieves the tetrahedral angle of $\sim 110^{\circ}$ when $r$ is $1.1 \AA$.

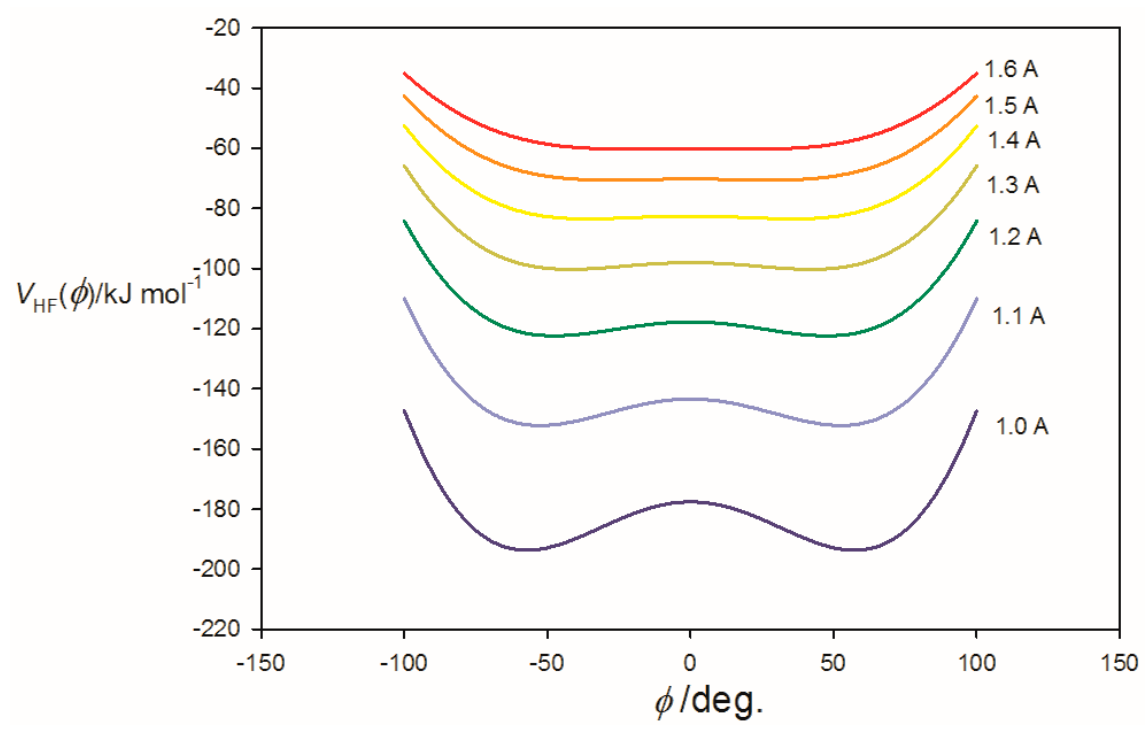

Figure 10. The electrostatic potential energy $V_{\mathrm{HF}}(\phi)$ of $\mathrm{HF}$ treated as a simple extended electric dipole as a function of angle $\phi$ at various distances of the $\mathrm{H}$ atom of $\mathrm{HF}$ from the $\mathrm{O}$ atom of 2,5-dihydrofuran.

The curves resulting when $V_{\mathrm{HF}}(\phi)$ is calculated as a function of angle $\phi$ at the three distances $r=1.5 \AA, 1.6 \AA$ and $1.7 \AA$ from $\mathrm{O}$ in oxetane and oxirane are shown in Figures 11 and 12, respectively. The three distances were chosen to straddle the experimental $[11,12,18,19]$ and the ab initio calculated lengths $r(\mathrm{O} \cdots \mathrm{H})$ (see Table 1 ) in each of the complexes oxetane $\cdots$ HF and oxirane $\cdots H F$. In the case of oxetane, we note that although the barrier height decreases as $r$ increases it remains quite high at between 10 and $20 \mathrm{~kJ} \mathrm{~mol}^{-1}$. Also $\phi_{\min }$ changes only slowly with $r$ and lies in the range $60^{\circ}$ to $65.5^{\circ}$. A similar situation obtains for oxirane (Figure 12), but now the barrier height ranges from 17 to $30 \mathrm{~kJ} \mathrm{~mol}^{-1}$ and $\phi_{\min }$ increases from $65^{\circ}$ to $69^{\circ}$ as $r$ decreases from 1.7 to $1.5 \AA$.

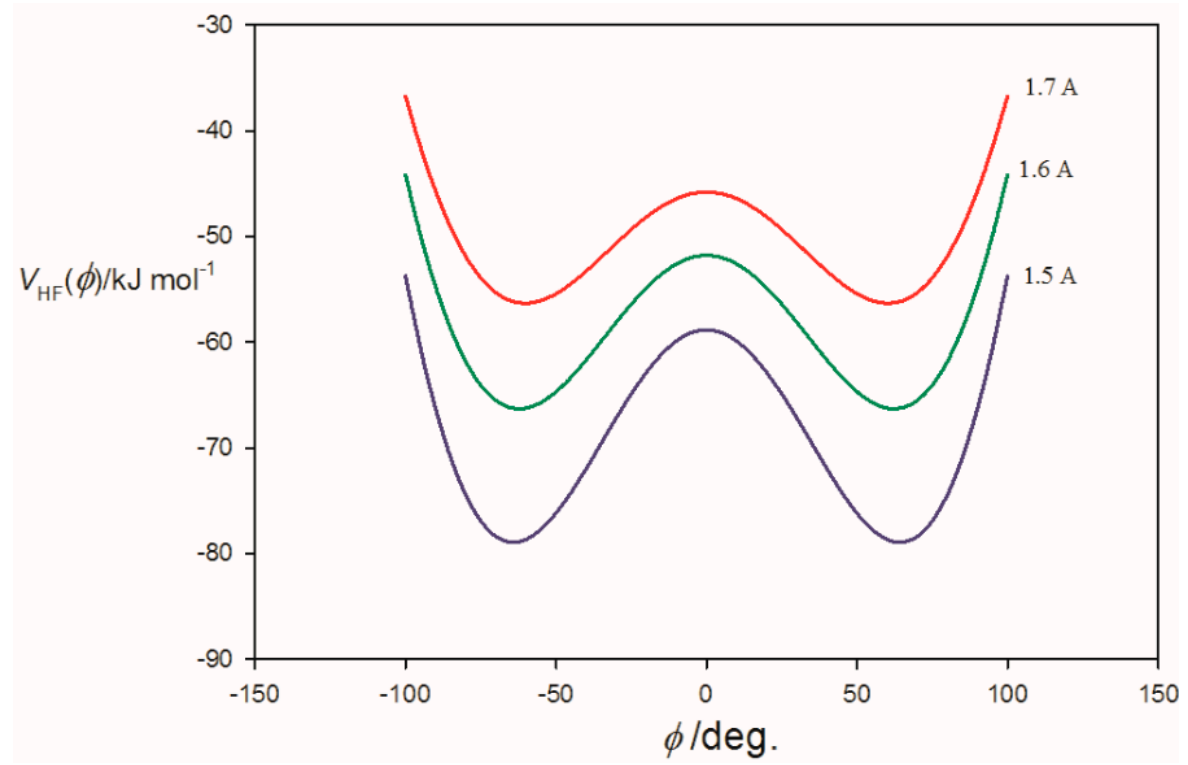

Figure 11. The electrostatic potential energy $V_{\mathrm{HF}}(\phi)$ of $\mathrm{HF}$ treated as a simple extended electric dipole as a function of angle $\phi$ at three distances of the $\mathrm{H}$ atom of $\mathrm{HF}$ from the $\mathrm{O}$ atom of oxetane. 


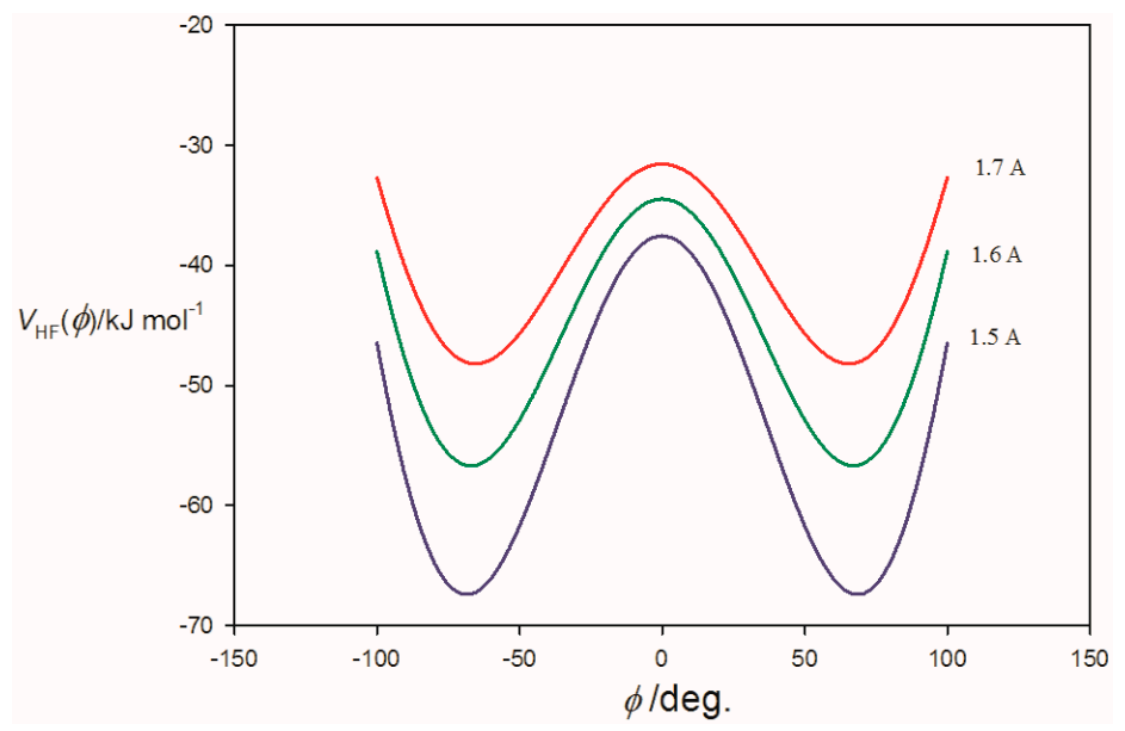

Figure 12. The electrostatic potential energy $V_{\mathrm{HF}}(\phi)$ of HF treated as a simple extended electric dipole as a function of angle $\phi$ at three distances of the $\mathrm{H}$ atom of $\mathrm{HF}$ from the $\mathrm{O}$ atom of oxirane.

Table 1. Comparison of properties of cyclic ether. . HF complexes from ab initio calculations, from rotational spectroscopy, and from an electrostatic model in which HF is treated as an extended electric dipole (see text for discussion of how $V_{\mathrm{HF}}(\phi)$ is calculated by using a DMA of the cyclic ether charge distribution in this case).

\begin{tabular}{cccc}
\hline Property & 2,5-Dihydrofuran $\cdots$ HF & Oxetane $\cdots$ HF & Oxirane $\cdots$ HF \\
\hline$r_{\mathrm{e}}(\mathrm{O} \cdots \mathrm{H}) / \AA$ & $1.631^{\mathrm{a}}$ & $1.622^{\mathrm{a}}$ & $1.663^{\mathrm{a}}$ \\
$r_{0}(\mathrm{O} \cdots \mathrm{H}) / \AA$ & $1.674^{\mathrm{b}}$ & $1.660^{\mathrm{c}}$ & $1.701^{\mathrm{d}}$ \\
$\phi_{\mathrm{e}} /^{\circ}$ & $48.24^{\mathrm{a}}$ & $57.36^{\mathrm{a}}$ & $73.08^{\mathrm{a}}$ \\
$\theta_{\mathrm{e}} /^{\circ}$ & 6.05 & 9.26 & 13.20 \\
$\phi_{0} /{ }^{\circ}$ & $46.3^{\mathrm{b}}$ & $57.9^{\mathrm{c}}$ & $72.0^{\mathrm{d}}$ \\
$\phi_{\min }\left(\text { at } r_{\mathrm{e}}\right)^{\mathrm{f}} /^{\circ}$ & $\sim 14$ & 62.0 & 66.0 \\
$\phi_{\min }\left(\text { at } r_{0}\right)^{\mathrm{g}} /{ }^{\circ}$ & $\sim 0$ & 61.0 & 65.5 \\
\hline
\end{tabular}

a Equilibrium values, calculated ab initio at the CCSD(T)-F12c/cc-pVTZ-F12 level of theory. ${ }^{\mathrm{b}}$ Ref. [11] The O $\cdots \mathrm{H}-\mathrm{F}$ nuclei were assumed collinear. ${ }^{c}$ Ref. [12] The $\mathrm{O} \cdots \mathrm{H}-\mathrm{F}$ nuclei were assumed collinear. ${ }^{\mathrm{d}}$ Ref. [13] The O $\cdots \mathrm{H}-\mathrm{F}$ nuclei were assumed collinear. ${ }^{\mathrm{e}} \theta_{\mathrm{e}}$ is the equilibrium value of the angular deviation of the $\mathrm{O} \cdots \mathrm{H}-\mathrm{F}$ nuclei from collinearity from the ab initio calculations. ${ }^{\mathrm{f}}$ Value of angle $\phi$ at the minimum of the potential energy curve $V_{\mathrm{HF}}(\phi)$ versus $\phi$ obtained when $\mathrm{HF}$ is treated as an extended electric dipole (see text for detail), the distance of the hydrogen bond $\mathrm{H}$ atom from $\mathrm{O}$ is taken as the the equilibrium value calculated ab initio, and the $\mathrm{O} \cdots \mathrm{H}-\mathrm{F}$ nuclei are constrained to be collinear. ${ }^{g}$ Value of angle $\phi$ at the minimum of the potential energy curve $V_{\mathrm{HF}}(\phi)$ versus $\phi$ obtained when HF is treated as an extended electric dipole (see text for detail), the distance of the hydrogen bond $\mathrm{H}$ atom from $\mathrm{O}$ is taken as the effective zero-point quantity from the analysis of the rotational spectrum, and the $\mathrm{O} \cdots \mathrm{H}-\mathrm{F}$ nuclei are constrained to be collinear.

Table 1 gives the values of $\phi_{\min }$ from the electrostatic potential energy functions $V_{\mathrm{HF}}(\phi)$ obtained by assuming the extended dipole moment model for HF in each of the three cyclic ether $\cdots \mathrm{HF}$ complexes. For comparison, Table 1 also includes (a) the corresponding experimental zero-point quantities $\phi_{0}$ (from rotational spectroscopy) and (b) the equilibrium counterparts $\phi_{\mathrm{e}}$ (from ab initio calculations at the CCSD(T)-F12c/cc-pVTZ-F12 level of theory). For convenience, the $\phi_{\min }$ are presented in two versions: one is calculated for the experimental zero-point distance $r_{0}(\mathrm{O} \cdots \mathrm{H})$ and the other at the ab initio equilibrium distance $r_{\mathrm{e}}(\mathrm{O} \cdots \mathrm{H})$. This is because the equilibrium distance is systematically shorter by $\sim 0.04 \AA$. Also included in Table 1 are the values of the equilibrium angular deviation $\theta$ of the $\mathrm{O} \cdots \mathrm{H}-\mathrm{F}$ nuclei from collinearity, as obtained from the ab initio calculations.

As expected from the preceding discussion of 2,5-dihydrofuran, the potential energy $V_{\mathrm{HF}}(\phi)$ of HF when treated as a simple extended electric dipole as a function of the angle $\phi$ significantly 
underestimates the angle $\phi_{\min }$ at the minimum (and indeed the barrier at the planar configuration at $\mathrm{O})$, for reasons discussed earlier. When the Lewis base is oxetane, $\phi_{\min }$ is found to be in reasonable agreement with the experimental and ab initio values of this quantity. For oxirane, $\phi_{\min }$ from the extended electric dipole model of HF appears to be a slight underestimate of both the zero-point and equilibrium values of $\phi$. It should be borne in mind that, unlike the extended electric dipole model in which the $\mathrm{O} \cdots \mathrm{H}-\mathrm{F}$ nuclei are held collinear, the hydrogen bond becomes increasingly non-linear along the series of HF complexed with 2,5-dihydrofuran, oxetane and oxirane. Nevertheless, the model does predict an increase in $\phi_{\min }$ as the OCO ring angle decreases from $\sim 108^{\circ}$, through $\sim 90^{\circ}$ to $\sim 60^{\circ}$ on replacing 2,5-dihydrofuran, by oxetane and then oxirane, and is qualitatively the order expected.

\section{Conclusions}

The electrostatic potential energy $V(\phi)$ of a non-perturbing unit positive charge has been calculated as a function of the angle $\phi$ at various fixed distances $r$ from the $\mathrm{O}$ atom in the three cyclic ethers 2,5-dihydrofuran, oxetane and oxirane ( $\phi$ is the angle made by the $r$ vector with the symmetry axis $C_{2}$ in each molecule). $V(\phi)$ was found to be a double-minimum function in each case, but the distance $r$ needs to be much shorter than the experimental distance $r(\mathrm{O} \cdots \mathrm{H})$ in the complex 2,5-dihydrofuran $\cdots \mathrm{HF}$ to produce a reasonable potential energy barrier to the planar configuration at $\mathrm{O}$. For oxetane and oxirane, double minima are apparent at the experimental distances $r(\mathrm{O} \cdots \mathrm{H})$ in the HF complexes of these cyclic ethers, but nevertheless the angles $\phi_{\min }$ at the minima are smaller than those $\phi_{0}$ and $\phi_{\mathrm{e}}$ found by experiment and by ab initio calculations, respectively, for these complexes. Reasons why $\phi_{\min }$ are underestimates and why the underestimates are more serious the smaller is the angle between the n-pairs carried by the $\mathrm{O}$ atom are presented.

A better locator of the directions of the n-pairs carried by $\mathrm{O}$ atoms of the cyclic ethers is the electrostatic potential energy $V_{\mathrm{HF}}(\phi)$ of the HF molecule as a function of $\phi$ when the diatomic molecule is modeled as a simple extended electric dipole. The results for $\phi_{\min }$ when using this model are in reasonable agreement with values from experiment and ab initio calculations and allow the conclusion that the corollary to the rules, namely that the HF molecule can be taken as a probe of n-pair directions, holds at least to a reasonable approximation. Thus, the angles between the n-pairs are in the order 2,5-dihydrofuran < oxetane < oxirane, a result consistent with the known increase in the ring angle OCO from $\sim 60^{\circ}$ in oxirane, through $\sim 90^{\circ}$ in oxetane to $\sim 108^{\circ}$ in 2,5-dihydrofuran.

Supplementary Materials: The following are available online at www.mdpi.com/2073-4352/7/9/261/s1. Optimised geometries of 2,5-dihydrofuran, oxetane and oxirane and of their hydrogen-bonded complexes with HF. Distributed multipole analyses of 2,5-dihydrofuran, oxetane and oxirane.

Author Contributions: J. Grant Hill and Anthony C. Legon conceived and carried out the calculations. J. Grant Hill and Anthony C. Legon analyzed the data and shared in the writing of the paper.

Conflicts of Interest: The authors declare no conflict of interest.

\section{References}

1. Arunan, E.; Desiraju, G.R.; Klein, R.A.; Sadlej, J.; Scheiner, S.; Alkorta, I.; Clary, D.C.; Crabtree, R.H.; Dannenberg, J.J.; Hobza, P.; et al. Defining the hydrogen bond: An account (IUPAC Technical Report). Pure Appl. Chem. 2011, 83, 1619-1636. [CrossRef]

2. Desiraju, G.R.; Shing Ho, P.; Kloo, L.; Legon, A.C.; Marquardt, R.; Metrangolo, P.; Politzer, P.A.; Resnati, G.; Rissanen, K. Definition of the halogen bond (IUPAC Recommendations 2013). Pure Appl. Chem. 2013, 85, 1711-1713. [CrossRef]

3. Legon, A.C. Tetrel, pnictogen and chalcogen bonds identified in the gas phase before they had names: A systematic look at non-covalent interactions. Phys. Chem. Chem. Phys. 2017, 19, 14884-14896. [CrossRef] [PubMed]

4. Clark, T.; Hennemann, M.; Murray, J.S.; Politzer, P.A. Halogen bonding: The $\sigma$-hole. J. Mol. Model. 2007, 13, 291-296. [CrossRef] [PubMed] 
5. Murray, J.S.; Lane, P.; Clark, T.; Riley, K.E.; Politzer, P.A. $\sigma$-Holes, $\pi$-holes and electrostatically-driven interactions. J. Mol. Model. 2012, 18, 541-548. [CrossRef] [PubMed]

6. Legon, A.C.; Millen, D.J. Determination of properties of hydrogen-bonded dimers by rotational spectroscopy and a classification of dimer geometries. Faraday Discuss. Chem. Soc. 1982, 73, 71-87. [CrossRef]

7. Legon, A.C.; Millen, D.J. Angular geometries and other properties of hydrogen-bonded dimers: A simple electrostatic interpretation based on the success of the electron-pair model. Chem. Soc. Rev. 1987, 16, 467-498. [CrossRef]

8. Buckingham, A.D.; Fowler, P.W. A model for the geometries of van der Waals complexes. Can. J. Chem. 1985, 63, 2018-2025. [CrossRef]

9. Kisiel, Z.; Legon, A.C.; Millen, D.J. Spectroscopic investigations of hydrogen bonding interactions in the gas phase. VII. The equilibrium conformation and out-of-plane bending potential energy function of the hydrogen-bonded heterodimer $\mathrm{H}_{2} \mathrm{O} \cdots \mathrm{HF}$ determined from its microwave rotational spectrum. Proc. R. Soc. Lond. A 1982, 381, 419-442. [CrossRef]

10. Guelachvili, G. Absolute wavenumber measurements of 1-0, 2-0, $\mathrm{HF}$ and $2-0 \mathrm{H}^{35} \mathrm{Cl}$ and $\mathrm{H}^{37} \mathrm{Cl}$ absorption bands. Opt. Commun. 1976, 19, 150-154. [CrossRef]

11. Georgiou, A.S.; Legon, A.C.; Millen, D.J. Spectroscopic investigations of hydrogen-bonding interactions in the gas phase. V. The identification and determination of the geometry and electric dipole moment of the hydrogen-bonded heterodimer formed between oxirane and hydrogen fluoride, $\left(\mathrm{CH}_{2}\right)_{2} \mathrm{O} \cdots \mathrm{HF}$, by infrared and microwave spectroscopy. Proc. R. Soc. Lond. A 1980, 373, 511-526.

12. Georgiou, A.S.; Legon, A.C.; Millen, D.J. Spectroscopic investigations of hydrogen bonding interactions in the gas phase. VI. A microwave spectroscopic determination of geometry for the heterodimer $\left(\mathrm{CH}_{2}\right)_{3} \mathrm{O} \cdots \mathrm{HF}$ formed between oxetane and hydrogen fluoride: Hydrogen bonding as a probe for lone pairs. J. Mol. Struct. 1980, 69, 69-78. [CrossRef]

13. Collins, R.A.; Millen, D.J.; Legon, A.C. Conformations of hydrogen-bonded dimers formed by cyclic ethers with hydrogen fluoride: Rotational spectrum of 2,5-dihydrofuran . H. HF. J. Mol. Struct. 1987, 162, 31-40. [CrossRef]

14. López, J.C.; Alonso, J.L.; Wlodarczak, G.; Demaison, J. The Millimeter-Wave Spectrum and ab Initio Calculations of 2,5-Dihydrofuran. J. Mol. Spectrosc. 1993, 160, 158-171. [CrossRef]

15. Creswell, R.A. Molecular structure of oxetane. Mol. Phys. 1975, 30, 217-222. [CrossRef]

16. Hirose, C. The microwave spectra and $r_{0}, r_{\mathrm{s}}$, and $r_{\mathrm{m}}$ structures of ethylene oxide. Bull. Chem. Soc. Jpn. 1974, 47, 1311-1318. [CrossRef]

17. López, J.C.; Blanco, S.; Lessari, A.; Sanz, M.E.; Lorenzo, F.J.; Alonso, J.L. HF inversion in the 2,5-dihydrofuran . . HF complex. J. Chem. Phys. 2001, 114, 9421-9429. [CrossRef]

18. Sanz, M.E.; Sanz, V.M.; López, J.C.; Alonso, J.L. Oxetane-hydrogen fluoride complex: A rotational study. Chem. Phys. Lett. 2001, 342, 32-38. [CrossRef]

19. Legon, A.C.; Wallwork, A.L.; Millen, D.J. Observation of $c$-type transitions and H,F nuclear spin-nuclear spin coupling in the rotational spectrum of $\left(\mathrm{CH}_{2}\right)_{2} \mathrm{O} \cdots \mathrm{HF}$. Chem. Phys. Lett. 1991, 178, 279-284. [CrossRef]

20. Clark, T.; Politzer, P.; Murray, J.S. Correct electrostatic treatment of noncovalent interactions: The importance of polarization. WIREs Comput. Mol. Sci. 2015, 5, 169-177. [CrossRef]

21. Wang, C.; Guan, L.; Danovich, D.; Shaik, S.; Mo, Y. The Origins of the Directionality of Noncovalent Intermolecular Interactions. J. Comput. Chem. 2016, 37, 34-45. [CrossRef] [PubMed]

22. Mustoe, C.L.; Gunabalasingam, M.; Yu, D.; Patrick, B.O.; Kennepohl, P. Probing covalency in halogen bonds through donor K-edge X-ray absorption spectroscopy: Polyhalides as coordination complexes. Faraday Discuss. 2017. [CrossRef] [PubMed]

23. Scheiner, $\mathrm{S}$. The Interplay between Charge Transfer, Rehybridization, and Atomic Charges in the Internal Geometry of Subunits in Noncovalent Interactions. Int. J. Quantum Chem. 2015, 115, 28-33. [CrossRef]

24. Kolár, M.H.; Hobza, P. Computer Modeling of Halogen Bonds and Other $\sigma$-Hole Interactions. Chem. Rev. 2016, 116, 5155-5187. [CrossRef] [PubMed]

25. Stone, A.J. Distributed multipole analysis, or how to describe a molecular charge distribution. Chem. Phys. Lett. 1981, 83, 233-239. [CrossRef]

26. Hättig, C.; Tew, D.P.; Köhn, A. Communications: Accurate and efficient approximations to explicitly correlated coupled-cluster singles and doubles, CCSD-F12. J. Chem. Phys. 2010, 132, 231102. [CrossRef] [PubMed] 
27. Werner, H.-J.; Knowles, P.J.; Knizia, G.; Manby, F.R.; Schutz, M.; Celani, P.; Gyorffy, W.; Kats, D.; Korona, T.; Lindh, R.; et al. Molpro, a Package of ab Initio Programs, Version 2015.1; University of Stuttgart: Stuttgart, Germany, 2015.

28. Werner, H.-J.; Knowles, P.J.; Knizia, G.; Manby, F.R.; Schütz, M. Molpro: A general-purpose quantum chemistry program package. Wiley Interdiscip. Rev. Comput. Mol. Sci. 2012, 2, 242-253. [CrossRef]

29. Peterson, K.A.; Adler, T.B.; Werner, H.-J. Systematically convergent basis sets for explicitly correlated wavefunctions: The atoms H, He, B-Ne, and Al-Ar. J. Chem. Phys. 2008, 128, 084102. [CrossRef] [PubMed]

30. Weigend, F. A fully direct RI-HF algorithm: Implementation, optimized auxiliary basis sets, demonstration of accuracy and efficiency. Phys. Chem. Chem. Phys. 2002, 4, 4285-4291. [CrossRef]

31. Kritikou, S.; Hill, J.G. Auxiliary Basis Sets for Density Fitting in Explicitly Correlated Calculations: The Atoms H-Ar. J. Chem. Theory Comput. 2015, 11, 5269-5276. [CrossRef] [PubMed]

32. Valeev, E.F. Improving on the resolution of the identity in linear R12 ab initio theories. Chem. Phys. Lett. 2004, 395, 190-195. [CrossRef]

33. Yousaf, K.E.; Peterson, K.A. Optimized auxiliary basis sets for explicitly correlated methods. J. Chem. Phys. 2008, 129, 184108. [CrossRef] [PubMed]

34. Dunning, T.H., Jr. Gaussian Basis Sets for Use in Correlated Molecular Calculations. I. The Atoms Boron through Neon and Hydrogen. J. Chem. Phys. 1989, 90, 1007-1023. [CrossRef]

35. Korona, T.; Moszynski, R.; Jeziorski, B. Electrostatic interactions between molecules from relaxed one-electron density matrices of the coupled cluster singles and doubles model. Mol. Phys. 2002, 100, 1723-1734. [CrossRef]

36. Krishnan, R.; Binkley, J.S.; Seeger, R.; Pople, J.A. Self-consistent molecular orbital methods. XX. A basis set for correlated wave functions. J. Chem. Phys. 1980, 72, 650-654. [CrossRef]

37. Deppmeier, B.J.; Driessen, A.J.; Hehre, T.S.; Hehre, W.J.; Johnson, J.A.; Klunzinger, P.E.; Leonard, J.M.; Pham, I.N.; Pietro, W.J.; Yu, J.; et al. SPARTAN'14 Mechanics Program: (Win/64b), Release 1.1.8, Wavefunction Inc.; Spartan Inc.: Bakersfield, CA, USA, 2014.

(C) 2017 by the authors. Licensee MDPI, Basel, Switzerland. This article is an open access article distributed under the terms and conditions of the Creative Commons Attribution (CC BY) license (http:/ / creativecommons.org/licenses/by/4.0/). 